\title{
Análises das Demandas e Cenários de Apoio para Sobreviventes de Câncer: Revisão Integrativa
}

doi: https://doi.org/10.32635/2176-9745.RBC.2021v67n4.1417

\author{
Analysis of the Demands and Scenarios of Support for Cancer Survivors: Integrative Review \\ Análisis de las Demandas y Escenarios de Apoyo para Sobrevivientes de Cáncer: Revisión Integradora
}

\author{
Andréia Rosangela Delli Paoli Rocha'; Erika Yuri Lee²; Heloisa Maria Jorviguinoviti dos Reis ; ${ }^{3}$ Edvane Birelo Lopes De Domenico4; \\ Maria das Graças Silva Matsubara ${ }^{5}$
}

\section{RESUMO}

Introduçáo: A sobrevivência do câncer é um processo que começa no diagnóstico e continua por toda a vida, trazendo uma série de desafios adicionais à saúde. Objetivo: Analisar as demandas e o modelo de atendimento para o apoio ao sobrevivente de câncer. Método: Revisão integrativa de literatura sobre demandas e cenários de apoio para sobreviventes de câncer, nas bases de dados LILACS, MEDLINE, IBECS, SciELO e Biblioteca Cochrane, que buscou artigos publicados entre janeiro de 2015 a março de 2020. Resultados: Foram encontrados 90 artigos, sendo 20 compatíveis com o objetivo do estudo. A estrutura necessária para apoiar o sobrevivente de câncer é múltipla e dela participam: hospitais, centros oncológicos e espaços na comunidade. Houve unanimidade em relação a intervenções clínicas e educativas solicitadas pelos pacientes. Estudos apontam atuação multidisciplinar, com presença de sintomas nos domínios físico, psicológico e socioecológico. A atuação do enfermeiro foi citada por 19 estudos e envolveu desde o cuidado clínico até a coordenação e atuaçáo quanto ao aconselhamento e orientação para a mudança de comportamentos prejudiciais à saúde. Verificaram-se oito estudos que citaram a American Society of Clinical Oncology como entidade norteadora das intervenções. Conclusáo: As demandas dos sobreviventes incluem acompanhamento clínico, apoio psicológico, orientaçôes de cuidados primários e estilo de vida saudável. As redes de apoio devem ser amplas e conduzidas principalmente por enfermeiros, embasadas em diretrizes sustentadas por evidências científicas e ou entidades especializadas com foco nos sintomas relacionados aos domínios físico, psicológico e socioecológico.

Palavras-chave: Oncologia; Sobreviventes de Câncer; Enfermagem Oncológica; Assistência Integral à Saúde; Literatura de Revisāo como Assunto.

\section{ABSTRACT}

Introduction: Cancer survival is a process beginning at diagnosis and continuing for life, bringing a series of additional health challenges. Objective: To analyze the demands and the care model to support cancer survivors. Method: Study of integrative literature review through search of articles published between January 2015 and March 2020 in the databases LILACS, MEDLINE, IBECS, SciELO and Cochrane Library about demands and support scenarios for cancer survivors. Results: Ninety articles were found, 20 of which are consistent with the objective of the study. The structure necessary to support the cancer survivor is multiple, and it includes: hospitals, cancer centers and spaces in the community. There was unanimity in meeting the needs of patients requiring clinical and educational interventions. Studies point out multidisciplinary performance, with the presence of symptoms in the physical, psychological, and socioecological domains. The role of the nurse was mentioned by 19 studies and involves clinical care up to coordination and initiatives related to counseling and guidance to change behaviors that are harmful to health. It was found that 8 studies cited the American Society of Clinical Oncology, as the guiding entity for the interventions. Conclusion: Survivors demands include clinical follow-up, psychological support, primary care guidelines and a healthy lifestyle. The support networks should be extensive, conducted mainly by nurses, based on guidelines supported by scientific evidence and/or specialized entities focused to symptoms related to the physical, psychological, and socioecological domains.

Key words: Medical Oncology; Cancer Survivors; Oncology Nursing; Comprehensive Health Care; Review Literature as Topic.

\section{RESUMEN}

Introducción: La supervivencia del cáncer es un proceso que comienza en el momento del diagnóstico y continúa durante toda la vida, lo que conlleva una serie de desafíos de salud adicionales. Objetivo: Analizar las demandas de necesidades y el modelo de atención para apoyar a los sobrevivientes de cáncer. Método: Estudio de revisión integradora de la literatura, sobre demandas y escenarios de apoyo para sobrevivientes de cáncer, en las bases de datos LILACS, MEDLINE, IBECS, SciELO y Cochrane Library, en la que se buscaron artículos publicados entre enero de 2015 y marzo de 2020. Resultados: Se encontraron noventa artículos, 20 de los cuales son consistentes con el objetivo del estudio. La estructura necesaria para apoyar al sobreviviente de cáncer es múltiple e incluye: hospitales, centros oncológicos y espacios en la comunidad. Hubo unanimidad en la satisfacción de las necesidades de los pacientes que requieren intervenciones clínicas y educativas. Los estudios apuntan a un desempeño multidisciplinario, con presencia de síntomas en los dominios físico, psicológico y socioecológico. El rol de la enfermera fue mencionado por 19 estudios, e incluye la atención clínica hasta la coordinación y desempeño en el asesoramiento y orientación para cambios de conducta que son perjudiciales para la salud. Se encontró que ocho estudios citaron a la American Society of Clinical Oncology, como entidad rectora de las intervenciones. Conclusión: Las demandas de los sobrevivientes incluyen seguimiento clínico, apoyo psicológico, pautas de atención primaria y un estilo de vida saludable. Las redes de apoyo deben ser desarrolladas de manera amplia principalmente por enfermeras, con base en pautas sustentadas en evidencia científica y/o entidades especializadas con foco en síntomas relacionados con los dominios físico, psicológico y socioecológico.

Palabras clave: Oncología Médica; Supervivientes de Cáncer; Enfermería Oncológica; Atención Integral de Salud; Literatura de Revisión como Asunto.

\footnotetext{
1,2,3Faculdade de Educação em Saúde Oswaldo Cruz. São Paulo (SP), Brasil. E-mails: andreiadelli@hotmail.com; enferikalee@gmail.com; helo_jviti@hotmail.com. Orcid iD: https://orcid.org/0000-0002-6147-8687; Orcid iD: https://orcid.org/0000-0001-7456-8350; Orcid iD: https://orcid.org/0000-0002-3519-0700

${ }^{4}$ Universidade Federal de São Paulo (Unifesp), Escola Paulista de Enfermagem (EPE), Departamento de Enfermagem Clínica e Cirúrgica. Acolhe-Onco. Grupo de Estudo em Prática e Educação Baseadas em Evidências (GEPEBE). São Paulo (SP), Brasil. E-mail: domenico.edvane@unifesp.br. Orcid iD: https://orcid.org/0000-00017455-1727

${ }^{5}$ Unifesp/EPE/GEPEBE. Hospital A. C. Camargo Cancer Center. Faculdade de Educação em Saúde Oswaldo Cruz. São Paulo (SP), Brasil. E-mail: maria.matsubara@accamargo.org.br. Orcid iD: https://orcid.org/0000-0002-9943-6722

Endereço para correspondência: Maria das Graças Silva Matsubara. Rua Paracatu, 553 apto. 124 - Parque Imperial. São Paulo (SP), Brasil. CEP 04002-021. E-mail maria.matsubara@accamargo.org.br
} 


\section{INTRODUÇÃO}

A incidência do câncer tem aumentado significativamente em todo o mundo, sendo considerada uma importante causa de morbidade e mortalidade, independente do índice de desenvolvimento humano. $\mathrm{O}$ risco cumulativo indica que um a cada oito homens e uma a cada dez mulheres desenvolveráo a doença ao longo da vida ${ }^{1}$.

No Brasil, as estimativas de câncer, para o triênio de 2020 e 2022, apontam a ocorrência de aproximadamente 625 mil novos casos de câncer para cada ano, com maior incidência para o câncer de pele náo melanoma (177 mil), seguido pelos cânceres de mama e próstata $(66 \mathrm{mil}$ cada), cólon e reto (41 mil), pulmão (30 mil) e estômago $(21 \mathrm{mil})^{2}$.

Essas estimativas refletem, proporcionalmente, na população de sobreviventes de acordo com a qualidade da política de controle e combate ao câncer ${ }^{3}$. Dados nacionais mostram que o número de sobreviventes tem aumentado, especialmente para os casos de câncer de mama e de próstata. Cerca de 206 milhóes de adultos sobreviveram ao câncer em 2012; destes, 23\% foram diagnosticados com câncer de mama e $20 \%$ com câncer de próstata ${ }^{4}$.

A justificativa para esse aumento relaciona-se com o envelhecimento demográfico, avanços na prevenção, detecção e tratamento, o que melhorou o prognóstico do câncer, com consequente aumento no número de pessoas com câncer ${ }^{5,6}$.

A sobrevivência do câncer é um processo que começa no diagnóstico e segue por toda a vida, trazendo uma série de desafios adicionais à saúde, pois pode ocorrer um câncer secundário ou outras comorbidades, problemas psicológicos, econômicos, além das toxicidades relacionadas ao tratamento, que envolvem também alteraçôes físicas ${ }^{7}$. Pode ser dividida em três fases: a aguda, se inicia no diagnóstico e termina com o tratamento inicial, tendo como ponto central o tratamento do câncer; a prolongada, origina-se no final do tratamento inicial e passa pelos meses seguintes, tendo como foco os efeitos do câncer e do tratamento; e a permanente, que constitui-se anos após o término do tratamento do câncer, sendo a fase com menor chance de recidiva 5 .

O conceito de sobrevivente do câncer, embora pouco conhecido no Brasil, traduz a complexidade do processo de adoecimento pelo câncer e a trajetória que advém de várias situaçóes e dificuldades constantes, envolvendo paciente e familiares, suscitando cuidado interdisciplinar para ambos ${ }^{7,8}$.

O término do tratamento do câncer gera ansiedade, sendo necessária sua adaptação, e o apoio dos profissionais da saúde é fundamental nessa etapa?
Para tanto, é importante um plano de cuidados para o sobrevivente de câncer, pois auxilia a coordenação da assistência pela equipe multidisciplinar, pelos próprios sobreviventes e outros envolvidos ${ }^{10}$.

Essa interaçáo entre profissional e paciente auxilia os sobreviventes a reconstruirem uma rotina de vida de acordo com suas necessidades e capacidades. A literatura relacionada à comunicaçáo no contexto do câncer indica que a relaçáo paciente e profissional é vital para a qualidade de vida $(\mathrm{QV})$ dos sobreviventes e um fator poderoso na qualidade dos cuidados?.

Nesse sentido, é preciso considerar que a sobrevivência ao câncer traz consigo uma série de desafios adicionais de saúde e de vida, como o aumento de risco para o desenvolvimento de cânceres secundários, possíveis comorbidades como diabetes, doenças cardíacas e osteoporose, alteraçôes psicológicas, o que requer atenção contínua e cuidados adequados ${ }^{11}$.

Portanto, por ser um processo complexo, tendo como foco a $\mathrm{QV}$, torna-se importante conhecer o impacto, possibilidades e o que considerar nessa fase, que possa ser fonte de suporte para o sobrevivente de câncer. Diante do exposto, este estudo tem como objetivo analisar as demandas, o modelo de atendimento, quanto às estruturas física e processual, para o apoio ao sobrevivente de diversos tipos de cânceres nos vários domínios, e os papéis desempenhados por profissionais, especialmente enfermeiro, nesse cenário.

\section{MÉTODO}

Trata-se de uma revisáo integrativa da literatura sobre as demandas e os cenários de apoio para sobreviventes de câncer.

O método empregado foi constituído por seis etapas: identificação do tema; elaboração das hipóteses ou questóes para a revisão integrativa; estabelecimento de critérios para inclusão e exclusão na literatura e seleção de amostra; apresentação das características dos estudos selecionados e análise crítica dos estudos incluídos; discussão dos resultados; apresentação da revisăo $0^{12,13}$.

Para tanto, as perguntas de estudo foram convertidas no acrônimo SPIDER ${ }^{14}$, conforme o Quadro 1.

Com base na estruturação das questôes e análise dos estudos, encaminharam-se as etapas subsequentes com a elaboração do Quadro 2. A partir dessa clareza intencional, procederam-se às etapas subsequentes. A escolha dos descritores foi realizada a partir da definição e análise da pertinência em relação ao tema da investigação, com base no Medical Subject Headings (MeSH) e nos descritores em Ciências da Saúde (DeCS), isolados e combinados: medical oncology; cancer survivors; oncology nursing; practice 
Quadro 1. Perguntas de estudo convertidas no acrônimo SPIDER

\begin{tabular}{|l|l|l|}
\hline S & SAMPLE ou amostra & Pacientes sobreviventes de câncer e enfermeiros \\
\hline PI & $\begin{array}{l}\text { Phenomenon of interest ou fenômeno } \\
\text { de interesse }\end{array}$ & $\begin{array}{l}\text { Principais resultados sobre as necessidades de cuidado dos } \\
\text { pacientes sobreviventes de câncer e estrutura necessária } \\
\text { para o atendimento dessas necessidades; papeis } \\
\text { desempenhados pelos enfermeiros }\end{array}$ \\
\hline D & Pesquisas de avaliação de QV & $\begin{array}{l}\text { Descritivo ou cluster de sinais e sintomas; estudos de } \\
\text { intervenção ou de relatos de processo de implantação de } \\
\text { assistência ao sobrevivente; estudos de enfermagem ou } \\
\text { interdisciplinar circunscritos aos sobreviventes de câncer; } \\
\text { publicados entre janeiro de 2015 e março de 2020, em } \\
\text { periódicos nacionais e internacionais (português, inglês e } \\
\text { espanhol) }\end{array}$ \\
\hline \multirow{2}{*}{ E } & Evaluation ou avaliação & $\begin{array}{l}\text { Resultados que caracterizam os fenômenos estudados: } \\
\text { sinais e sintomas na sobrevivência ao câncer, organização } \\
\text { física e processual para o cuidado e as atividades dos } \\
\text { enfermeiros }\end{array}$ \\
\hline R & Research type ou tipos de pesquisas & \begin{tabular}{l} 
Métodos quantitativos ou mistos \\
\hline
\end{tabular}
\end{tabular}

guidelines as topic; comprehensive health care, nas bases de dados e ou bibliotecas eletrônicas LILACS, MEDLINE (PubMed), IBECS, SciELO e Biblioteca Cochrane.

O processo de seleção dos artigos foi realizado por dois pesquisadores, que o fizeram de maneira independente, e as análises foram comparadas posteriormente. $\mathrm{Na}$ divergência, um terceiro pesquisador foi envolvido para a tomada de decisão ${ }^{15}$.

\section{RESULTADOS}

Foram identificados, inicialmente, 90 artigos distribuídos pelas bases de dados citadas, que atendiam ao tema proposto. No entanto, ao aplicar os critérios de elegibilidade, foram selecionados 20 artigos, publicados nos últimos cinco anos, sendo $85 \%$ destes na língua inglesa. A Figura 1 exemplifica o processo de revisão de literatura e estudo de caso.

No Quadro 2, foi realizada uma síntese dos artigos antes indicados e analisados $s^{8,16-33}$. Identificou-se o predomínio de pesquisas de níveis de evidência 4 (80\%), com publicaçóes distribuídas dentro do período do estudo, porém, com hegemonia no ano de 2015, perfazendo $30 \%$ dos estudos. O conjunto das variáveis investigadas foi amplo, oferecendo possibilidade de análise sobre os fatores intervenientes quanto às demandas e cenários de apoio aos sobreviventes de câncer.

\section{DISCUSSÃO}

Observa-se que as demandas dos sobreviventes envolvem acompanhamento clínico, orientaçóes de cuidados primários, aconselhamento sexual e de fertilidade, além do estilo de vida saudável e apoio psicológico. Nesse sentido, a American Society of Clinical Oncology (ASCO) ${ }^{25}$ divulgou diretrizes de prática clínica baseadas em evidências direcionadas para a prevenção e gerenciamento de sintomas que afetam muitos sobreviventes, que apresentam efeitos tardios. As recomendações reforçam a atenção às necessidades físicas e psicológicas dos sobreviventes do câncer ${ }^{25}$.

$\mathrm{O}$ atendimento dessa população requer uma estrutura física e processual, que pode ser de forma invidualizada

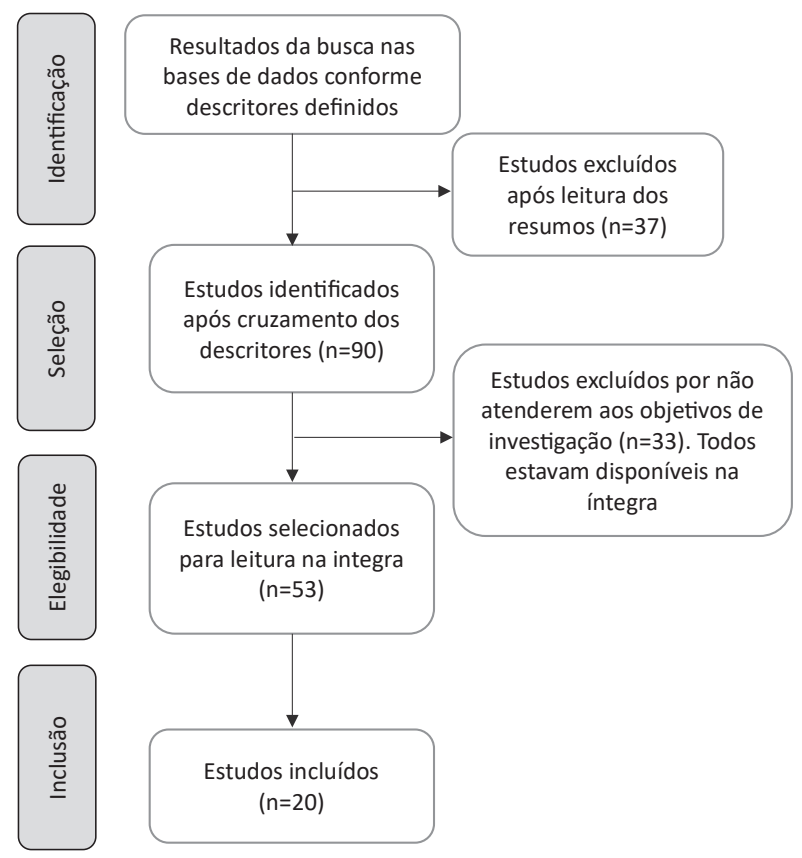

Figura 1. Representação das etapas de elegibilidade dos artigos científicos 
Quadro 2. Apresentação dos estudos utilizados

\begin{tabular}{|c|c|c|c|}
\hline Autor/ano & Objetivos & $\begin{array}{l}\text { Método/Nível de } \\
\text { evidência }\end{array}$ & Principais achados \\
\hline $\begin{array}{l}\text { Oliveira et } \\
\text { al., } 2017^{8}\end{array}$ & $\begin{array}{l}\text { Analisar relatos, por meio de entrevistas } \\
\text { semiestruturadas com sobreviventes de } \\
\text { câncer, sobre a interação com a equipe } \\
\text { de saúde e descrever semelhanças e } \\
\text { diferenças nos padrões de interação e } \\
\text { cuidado de saúde brasileira e canadense }\end{array}$ & $\begin{array}{l}\text { Estudo de casos com } \\
\text { abordagem qualitativa } \\
\text { descritiva interpretativa } \\
\text { NE: } 3\end{array}$ & $\begin{array}{l}\text { Consultas ambulatoriais em hospitais e orientação por equipe de médicos } \\
\text { e enfermeiros (telenursing e telemedicina). Indicação de sites e livros para } \\
\text { informação. Os sintomas incluem: insegurança, depressão e disfunção } \\
\text { sexual. Realização de consultas e orientaçôes por médicos e enfermeiros. } \\
\text { Enfermeiro assume cuidados clínicos e tem um papel importante para } \\
\text { uma boa comunicação com os pacientes, com câncer urológico (população } \\
\text { masculina) }\end{array}$ \\
\hline $\begin{array}{l}\text { Wallace et } \\
\text { al., } 2015^{16}\end{array}$ & $\begin{array}{l}\text { Examinar as perspectivas dos enfermeiros } \\
\text { oncológicos sobre a rede de atendimento } \\
\text { na assistência aos sobreviventes; contribuir } \\
\text { para um estudo maior que trata da } \\
\text { inclusão na rotina da enfermagem de } \\
\text { cuidados com sobreviventes de câncer }\end{array}$ & $\begin{array}{l}\text { Pesquisa transversal } \\
\text { quantitativa, com } 119 \\
\text { enfermeiros que cuidam } \\
\text { de pacientes com câncer } \\
\text { hematológico } \\
\text { NE: } 4\end{array}$ & $\begin{array}{l}\text { Implantação de grupos de apoio em Centros de Câncer, para discussão } \\
\text { e orientação sobre fertilidade, intimidade e sexualidade, além de } \\
\text { orientação sobre cuidados primários, realizado em ambulatórios. Os } \\
\text { sintomas incluem: limitações financeiras, psicológicas e sociais, depressão, } \\
\text { infertilidade e disfunção sexual. Cuidados técnicos e clínicos prestados por } \\
\text { enfermeiros, envolvendo linfomas e leucemias }\end{array}$ \\
\hline $\begin{array}{l}\text { Cox et al., } \\
2015^{17}\end{array}$ & $\begin{array}{l}\text { Acompanhar visões e experiências } \\
\text { de mulheres sobreviventes do câncer } \\
\text { de ovário; obter informações sobre } 0 \\
\text { processo de enfrentamento e contribuir } \\
\text { para desenvolver abordagens inovadoras } \\
\text { centradas no atendimento dos } \\
\text { sobreviventes }\end{array}$ & $\begin{array}{l}\text { Estudo qualitativo com } \\
\text { análise fenomenológica } \\
\text { interpretativa } \\
\text { NE: } 4\end{array}$ & $\begin{array}{l}\text { Grupos de apoio, com acompanhamento clínico telefônico por } 3 \text { anos. } \\
\text { Morbidade psicossocial e necessidade de ajuste físico. Atuação do } \\
\text { enfermeiro no domínio emocional com orientação psicossocial e sobre os } \\
\text { exames de vigilância, para pacientes com câncer de ovário }\end{array}$ \\
\hline $\begin{array}{l}\text { Hall-Alston, } \\
2015^{18}\end{array}$ & $\begin{array}{l}\text { Mostrar as implicações da rotina de } \\
\text { exerć́cios na trajetória de sobrevivência } \\
\text { ao câncer }\end{array}$ & $\begin{array}{l}\text { Revisão bibliográfica } \\
\text { NE: } 4\end{array}$ & $\begin{array}{l}\text { Grupos de apoio, com uso de salas e ambulatórios para prática de } \\
\text { exercícios físicos, embasados nas diretrizes da ASCO. Os sintomas incluem: } \\
\text { ansiedade, depressão, medo da recorrência do câncer, aumento do } \\
\text { risco de doenças crônicas e mudança do corpo com a retirada da mama. } \\
\text { Enfermeiros organizam ambientes para promoção de exerćícios físicos, } \\
\text { com foco em câncer de mama }\end{array}$ \\
\hline $\begin{array}{l}\text { Campbell et } \\
\text { al., 201919 }\end{array}$ & $\begin{array}{l}\text { Definir aspectos importantes para cuidado } \\
\text { das sobreviventes de câncer ginecológico } \\
\text { nos EUA; avaliar e gerenciar os cuidados } \\
\text { para qualidade de vida das sobreviventes }\end{array}$ & $\begin{array}{l}\text { Revisão bibliográfica } \\
\text { NE: } 4\end{array}$ & $\begin{array}{l}\text { Grupos de apoio, realizados em ambulatórios e Centros de Câncer, } \\
\text { embasados nas diretrizes da Society of Gynecology Oncology. Problemas } \\
\text { observados: sexuais, depressão, incontinência urinária, dor, distúrbios } \\
\text { alimentares e no sono. Atuaçãa do enfermeiro com programa educativo, } \\
\text { coordenação médica, orientação nutricional e recomendaçóes de exercícios } \\
\text { físicos, com foco em câncer ginecológico (cervical, endométrio ovário e } \\
\text { vulva) }\end{array}$ \\
\hline $\begin{array}{l}\text { Heveran e } \\
\text { Andrewes, } \\
2020^{20}\end{array}$ & $\begin{array}{l}\text { Rever necessidades de apoio aos } \\
\text { sobreviventes de câncer hematológico; } \\
\text { estabelecer medidas de enfermagem para } \\
\text { suporte }\end{array}$ & $\begin{array}{l}\text { Revisão de literatura } \\
\text { e análise temática de } \\
\text { fontes primárias } \\
\text { NE: } 4\end{array}$ & $\begin{array}{l}\text { Necessidade de rede de apoio voltada à informação, tratamento físico } \\
\text { e psicológico. Educação com uso do plano de sobrevivência contendo } \\
\text { direcionamento sobre: atividades físicas, nutrição, exames regulares, } \\
\text { necessidade de suporte na área de enfermagem de forma individualizada } \\
\text { e em grupos de apoio. Os sintomas incluem: depressão, ansiedade, } \\
\text { infecções, fadiga, dificuldade respiratória, problemas cardiovasculares, } \\
\text { problema com fertilidade e sensação de abandono. Ação do enfermeiro } \\
\text { especialista no atendimento clínico e educacional para pacientes com } \\
\text { linfomas e leucemias }\end{array}$ \\
\hline $\begin{array}{l}\text { Karvinen et } \\
\text { al., } 2015^{21}\end{array}$ & $\begin{array}{l}\text { Examinar as práticas de aconselhamento } \\
\text { sobre comportamento no estilo de vida de } \\
\text { enfermeiros oncológicos aos sobreviventes } \\
\text { de câncer }\end{array}$ & $\begin{array}{l}\text { Estudo quantitativo } \\
\text { NE: } 4\end{array}$ & $\begin{array}{l}\text { Atendimento clínico ambulatorial, para aconselhamento sobre estilo } \\
\text { de vida: nutrição, prática de atividades físicas. Atendimento individual } \\
\text { presencial com base no CANO-ACIO. Problemas psicológicos (depressão } \\
\text { e ansiedade), sociais e financeiros. Enfermeiros oncológicos: atuação } \\
\text { clínica e participação no programa educativo de aconselhamento } \\
\text { comportamental, com foco nos diversos tipos de cânceres }\end{array}$ \\
\hline
\end{tabular}


Quadro 2. continuação

\begin{tabular}{|c|c|c|c|}
\hline Autor/ano & Objetivos & $\begin{array}{c}\text { Método/Nível de } \\
\text { evidência }\end{array}$ & Principais achados \\
\hline $\begin{array}{l}\text { Ferguson } \\
\text { e Aning, } \\
2015^{22}\end{array}$ & $\begin{array}{l}\text { Descrever a implementação de programa } \\
\text { de sobrevivência para atendimento a } \\
\text { sobreviventes de câncer de próstata }\end{array}$ & $\begin{array}{l}\text { Estudo descritivo sobre } \\
\text { implementação de } \\
\text { modelo de atendimento } \\
\text { a sobreviventes de } \\
\text { câncer de próstata } \\
\text { NE: } 4\end{array}$ & $\begin{array}{l}\text { Grupo de apoio, realizado em ambulatório e atendimento individualizado, } \\
\text { com base no plano elaborado pela instituição: Newcastle upon Tyne } \\
\text { Hospitals NHS Foundation Trust. Sintomas incluem: disfunção erétil, } \\
\text { ondas de calor e frustração. Atuação do enfermeiro no âmbito clínico } \\
\text { e educacional com aconselhamento sobre exercícios físicos, orientação } \\
\text { psicossocial e psicossexual, para paciente com câncer de próstata }\end{array}$ \\
\hline $\begin{array}{l}\text { Singh- } \\
\text { Carlson et } \\
\text { al., } 2018^{23}\end{array}$ & $\begin{array}{l}\text { Avaliar o desenvolvimento e } \\
\text { implementação de um plano de atenção } \\
\text { à sobrevivência ao câncer de mama no } \\
\text { Sul da Ásia }\end{array}$ & $\begin{array}{l}\text { Abordagem longitudinal } \\
\text { de métodos mistos } \\
\text { (qualitativo e } \\
\text { quantitativo) } \\
\mathrm{NE}: 4\end{array}$ & $\begin{array}{l}\text { Grupo de apoio, realizado em ambulatório e atendimento individualizado, } \\
\text { por meio de aconselhamento de pacientes e familiares com uso de } \\
\text { questionários via e-mail. Os sintomas incluem: depressão, ansiedade, } \\
\text { dificuldades psicossociais em relação à sexualidade e medo da } \\
\text { reincidência. Atuação de enfermeiros e médicos oncológicos. Enfermeiros } \\
\text { atuam no atendimento clínico e educativo com foco na orientação sobre } \\
\text { necessidades físicas, sintomas psicossociais, sexualidade e estilo de vida, } \\
\text { com foco no câncer de mama }\end{array}$ \\
\hline $\begin{array}{l}\text { Nolte et al., } \\
2016^{24}\end{array}$ & $\begin{array}{l}\text { Examinar o impacto em pacientes e } \\
\text { profissionais de saúde da implementação } \\
\text { do SCP em um Centro de Câncer da } \\
\text { Austrália }\end{array}$ & $\begin{array}{l}\text { Estudo de caso, com uso } \\
\text { de questionário } \\
\mathrm{NE}: 4\end{array}$ & $\begin{array}{l}\text { Follow-up com pacientes para suporte pós-tratamento, por meio de grupos } \\
\text { de apoio, com atendimento ambulatorial, baseado nas diretrizes da ASCO } \\
\text { e IOM. Problemas psicossociais e físicos. Atuação de enfermeiros e médicos } \\
\text { com base no SCP, para cânceres: ginecológicos, hematológico e urológicos }\end{array}$ \\
\hline $\begin{array}{l}\text { Corcoran et } \\
\text { al., } 2015^{25}\end{array}$ & $\begin{array}{l}\text { Revisar a prática avançada da } \\
\text { enfermagem no planejamento, } \\
\text { implementação e avaliação do cuidado dos } \\
\text { sobreviventes do câncer }\end{array}$ & $\begin{array}{l}\text { Revisão de literatura } \\
\text { NE:4 }\end{array}$ & $\begin{array}{l}\text { Grupos de apoio realizados em centros clínicos com ambulatórios para } \\
\text { atendimento oncológico, por meio de reunião de grupos e atendimento } \\
\text { individual, baseado nas diretrizes da ASCO. Os sintomas incluem: } \\
\text { disfunção sexual, dor, fadiga e estresse psicológico. Atuação da equipe } \\
\text { multidisciplinar, com coordenação de enfermeiros. Avaliação psicossocial, } \\
\text { serviços de nutrição, atividade física e suporte a familiares. Enfermeiros } \\
\text { especialistas atuam no planejamento, implementação de programas } \\
\text { educativos em saúde e cuidados clínicos, para pacientes com todos os tipos } \\
\text { de cânceres }\end{array}$ \\
\hline $\begin{array}{l}\text { Peixoto et } \\
\text { al., } 2017^{26}\end{array}$ & $\begin{array}{l}\text { Conhecer estratégias de gestão de } \\
\text { ansiedade utilizadas pelos sobreviventes } \\
\text { de câncer }\end{array}$ & $\begin{array}{l}\text { Estudo de revisão } \\
\text { sistemática de literatura } \\
\text { NE: } 2\end{array}$ & $\begin{array}{l}\text { Grupos de apoio e terapia em grupo com atendimento ambulatorial } \\
\text { focado na reabilitação. Os sintomas incluem: ansiedade, depressão, } \\
\text { estresse e fadiga. Atuação da equipe multidisciplinar (enfermeiros, } \\
\text { terapeutas ocupacionais e médicos), com gestão dos processos de saúde- } \\
\text {-doença, reabilitação e intervenção piscoeducacional. Enfermeiro com } \\
\text { atuação em atendimento clínico, assistencial e indicação de prática de } \\
\text { exerćicios físicos, com predomínio do câncer de mama }\end{array}$ \\
\hline $\begin{array}{l}\text { Oliveira et } \\
\text { al., } 2018^{27}\end{array}$ & $\begin{array}{l}\text { Analisar o conceito de sobrevivência do } \\
\text { câncer usando o modelo de análise de } \\
\text { conceito evolutivo de Rodgers }\end{array}$ & $\begin{array}{l}\text { Revisão sistemática de } \\
\text { literatura, com uso do } \\
\text { conceito de Rodgers } \\
\text { NE: } 2\end{array}$ & $\begin{array}{l}\text { Grupos de apoio para monitorar a qualidade de vida dos sobreviventes, } \\
\text { realizados em salas nas comunidades e centros clínicos. Problemas físicos, } \\
\text { psicológicos e financeiros. Atuação equipe multidisciplinar (educador físico, } \\
\text { psicólogo e assistente social), para monitorar a qualidade de vida de } \\
\text { pacientes com todos os tipos de cânceres }\end{array}$ \\
\hline $\begin{array}{l}\text { Loonen et } \\
\text { al., } 2018^{28}\end{array}$ & $\begin{array}{l}\text { Apresentar modelos de atendimento } \\
\text { multidisciplinar e cuidados compartilhados } \\
\text { para os sobreviventes de câncer }\end{array}$ & $\begin{array}{l}\text { Estudo descritivo } \\
\text { NE: } 4\end{array}$ & $\begin{array}{l}\text { Grupos de apoio, com atendimento em clínicas especializadas para } \\
\text { sobreviventes de câncer, baseados em plano da própria instituiçãa. } \\
\text { Problemas com sexualidade, depressão, ansiedade e limitações físicas. } \\
\text { Atuação de médico, psicólogo, assistente social, enfermeiro, dentista e } \\
\text { nutricionista, com programa educativo sobre atividade física, nutrição, } \\
\text { sexualidade e cuidados clínicos. Enfermeiro assume a coordenação das } \\
\text { equipes, com foco nos diversos tipos de cânceres em adultos e crianças }\end{array}$ \\
\hline
\end{tabular}


Quadro 2. continuação

\begin{tabular}{|c|c|c|c|}
\hline Autor/ano & Objetivos & $\begin{array}{l}\text { Método/Nível de } \\
\text { evidência }\end{array}$ & Principais achados \\
\hline $\begin{array}{l}\text { Kinahan et } \\
\text { al., } 2017^{29}\end{array}$ & $\begin{array}{l}\text { Avaliar a implementação de boas práticas } \\
\text { para aumentar as informações nos } \\
\text { prontuários eletrônicos aos prestadores } \\
\text { de cuidados primários (médicos } \\
\text { e enfermeiros) para atendimento } \\
\text { compartilhado de jovens e adultos } \\
\text { sobreviventes de câncer }\end{array}$ & $\begin{array}{l}\text { Estudo de casos, com } \\
\text { abordagem qualitativa } \\
\text { NE: } 4\end{array}$ & $\begin{array}{l}\text { Grupos de apoio e atendimento individual em ambulatório e Centros } \\
\text { de Câncer, com acompanhamento dos casos por meio de controle de } \\
\text { prontuário eletrônico para possíveis intervenções, com base nas diretrizes } \\
\text { da ASCO. Os problemas incluem: depressão, ansiedade, sexualidade e } \\
\text { financeiros. Atuação multidisciplinar para pacientes com cânceres em } \\
\text { geral, com foco na nutrição, exerćicios físicos e sexualidade }\end{array}$ \\
\hline $\begin{array}{l}\text { Peckham e } \\
\text { Mott-Coles, } \\
2018^{30}\end{array}$ & $\begin{array}{l}\text { Determinar as contribuições do enfermeiro } \\
\text { especialista em Oncologia no atendimento } \\
\text { ao paciente em monitoramento } \\
\text { radiológico, após o diagnóstico precoce de } \\
\text { câncer de pulmão }\end{array}$ & $\begin{array}{l}\text { Estudo descritivo } \\
\text { e retrospectivo } \\
\text { de gráficos de } \\
\text { sobreviventes de } \\
\text { câncer de pulmão } \\
\text { NE: } 4\end{array}$ & $\begin{array}{l}\text { Grupos de apoio com atendimento individualizado em hospitais e Centros } \\
\text { de Câncer, baseados nas diretrizes da ASCO. Dificuldades respiratórias. } \\
\text { Atuação do enfermeiro oncológico como facilitador na comunicação com } \\
\text { paciente e famílias, com foco no câncer de pulmão }\end{array}$ \\
\hline $\begin{array}{l}\text { Handberg ef } \\
\text { al., } 2018^{31}\end{array}$ & $\begin{array}{l}\text { Analisar e descrever as atitudes e } \\
\text { perspectivas dos profissionais de saúde } \\
\text { sobre as complexidades da sobrevivência } \\
\text { ao câncer }\end{array}$ & $\begin{array}{l}\text { Estudo descritivo e } \\
\text { interpretativo } \\
\text { NE: } 4\end{array}$ & $\begin{array}{l}\text { Atendimento ambulatorial e grupos de apoio, em Centros de Câncer } \\
\text { e ambulatório, segundo as diretrizes da ASCO. Os sintomas incluem: } \\
\text { ansiedade, fadiga, depressão e preocupação com os familiares. } 0 \\
\text { enfermeiro oncológico é responsável em estabelecer o elo entre o paciente } \\
\text { e o responsável pelo tratamento do câncer, prestando cuidados clínicos e } \\
\text { orientação quanto à saúde, para pacientes com câncer hematológico }\end{array}$ \\
\hline $\begin{array}{l}\text { Pandey } \\
\text { e Barber, } \\
2016^{32}\end{array}$ & $\begin{array}{l}\text { Descrever a falta de profissionais para } \\
\text { cuidados básicos e médicos oncológicos } \\
\text { nos Centro de Câncer dos EUA; discutir } \\
\text { a necessidade de treinamento dos } \\
\text { enfermeiros para especialização em } \\
\text { Oncologia e cuidados básicos aos } \\
\text { sobreviventes }\end{array}$ & $\begin{array}{l}\text { Estudo de caso } \\
\text { NE: } 4\end{array}$ & $\begin{array}{l}\text { Grupos de apoio, com atendimento em grupo ou individual em clínica } \\
\text { de cuidados primários e ambulatório, com base nas dirtrizes da ASCO. } 0 \\
\text { sintoma inclui a dor. A atuação do enfermeiro visa à prevenção de câncer } \\
\text { secundário, aconselhamento em saúde e programas educativos, com } \\
\text { orientação sobre cessação do tabagismo, nutrição e exerćcios físicos, para } \\
\text { diversos tipos de cânceres }\end{array}$ \\
\hline $\begin{array}{l}\text { Hartneft, } \\
2016^{33}\end{array}$ & $\begin{array}{l}\text { Discutir as recomendações da ACS para } \\
\text { vigilância, triagem e gestão dos efeitos } \\
\text { tardios do tratamento para câncer de } \\
\text { mama }\end{array}$ & $\begin{array}{l}\text { Estudo descritivo } \\
\mathrm{NE}: 4\end{array}$ & $\begin{array}{l}\text { Grupos de apoio, em ambulatórios e Centros de Câncer, baseados } \\
\text { nas diretrizes da ASCO. Os sintomas incluem: depressão, ansiedade } \\
\text { e problemas nas relações sexuais. Atuação da equipe multidisciplinar } \\
\text { (enfermeiro e terapeuta ocupacional), conduzindo reuniões, para } \\
\text { orientação sobre nutrição, exerćícios físicos e sexualidade. Enfermeiros } \\
\text { fornecem informações educacionais para auxiliar no seguimento das } \\
\text { diretrizes após o tratamento, para pacientes com câncer de mama }\end{array}$ \\
\hline
\end{tabular}

Legendas: $\mathrm{ASCO}=$ American Society of Clinical Oncology; $\mathrm{SCP}=$ Survivorship Care Plan; $\mathrm{CANO}-\mathrm{ACIO}=$ Canadian Association of Nurses in Oncology; $\mathrm{ACS}=$ American Cancer Society; IOM = Institute of Medicine; NE = Nível de evidência.

ou em grupo, com base nas diretrizes desenvolvidas por organizaçōes profissionais ou pela própria instituição de saúde, realizadas em centros oncológicos, unidades ambulatoriais, situadas dentro de hospitais ou em salas disponíveis na comunidade. Muitos hospitais na Europa, EUA, Canadá e Austrália estabelecem clínicas ou os chamados Centros de Câncer com ambulatórios e salas para o atendimento dos sobreviventes de câncer ${ }^{28}$.

No mesmo contexto, a American Cancer Society (ACS) estabeleceu recursos financeiros para a criaçáo de Centros Clínicos para cuidados do câncer, voltados para os sobreviventes ${ }^{25}$. A ASCO também se envolveu com esse tema, criando em 2012 um Comitê de Sobrevivência, com agenda abrangente para o desenvolvimento de centros para cuidados de sobreviventes, com efeitos de longo prazo ${ }^{25}$.

Organizaçōes, como o Institute of Medicine (IOM), a Comissão Americana de Cirurgióes do Câncer do American College, a National Coalition for Cancer Survivorship (NCCS) e a ASCO se envolveram com o tema sobrevivência, tendo como recomendação a implementação de planos de cuidados que envolvem diretrizes para a assistência ao sobrevivente, com objetivos de melhorar a vigilância, a qualidade assistencial após o tratamento e cuidados relacionados à saúde. Esses planos incluem o resumo do tratamento, os efeitos colaterais potenciais, as diretrizes de vigilância, cuidados de acompanhamento, avisos de saúde e problemas que afetam a $\mathrm{QV}^{34,35}$. 
Quanto aos sintomas, relacionam-se aos domínios físico, psicolólogico e socioecológico, os quais incluem depressão, ansiedade, medo de recidiva, disfunção sexual, infertilidade, dor, fadiga, incontinência urinária, problemas respiratórios, ondas de calor, distúrbio do sono e alimentar. Além do diagnóstico, as diversas modalidades de tratamento também geram problemas físicos, sociais e psicológicos, com repercussão na $\mathrm{QV}$, e podem persistir ao longo da sobrevida ${ }^{36,37}$. Ou seja, tanto o diagnóstico quanto o tratamento do câncer estão associados a um sofrimento substancial, dificultando o retorno a vida normal $^{37-39}$.

Outro ponto de preocupação diz respeito ao aumento da probabilidade de doenças crônicas, comorbidades e a recorrência de cânceres secundários, mediante ausência de prática de comportamentos saudáveis, como a falta de atividade física e o uso do tabaco, o que impacta na $\mathrm{QV}^{21}$.

Em relação aos papéis desempenhados por profissionais, observa-se a atuação da equipe multidisciplinar, seja nos cuidados clínicos ou programas educativos, com orientaçôes psicossiocial, nutricional, psicosexual, exames de vigilância, prática de exercícios e necessidades físicas. A diversidade de alteraçóes relacionadas aos diversos domínios, indica a necessidade de acompanhamento multidisciplinar ${ }^{19}$. Observam-se depressão, ansiedade e medo de recidivas quando não existe acompanhamento no período pós-tratamento ${ }^{20}$. Esses sintomas podem ser atenuados com orientação e atendimento psicossociais adequados ${ }^{21}$.

Observa-se atuação ativa de enfermeiros, seja nos cuidados clínicos ou planejamento e implementação de modelos de atendimento com programas educativos em saúde. $\mathrm{O}$ fato de os enfermeiros constituírem maior grupo de profissionais entre as demais categorias, além da interaçáo com os pacientes no processo de saúde-doença, resulta em um papel de destaque na asssitência ao sobrevivente de câncer ${ }^{26}$. Além do cuidado clínico, esse profissional pode assumir papel de educador sobre programas em saúde, dietas saudáveis e prática de exercícios físicos, assim como a posição de gestão, desenvolvendo e avaliando modelos de cuidados para uma variedade de configuraçóes práticas, o que reflete na $\mathrm{QV}$ do sobrevivente de câncer ${ }^{20,40}$.

Também ressalta-se a atuação ativa da Oncology Nursing Society (ONS), na reabilitaçáo e QV dos sobrevivente do câncer, desenvolvendo recursos baseados em evidências para orientar o ensino e intervençóes de atendimento a essa população $0^{25}$.

Um estudo realizado na Holanda descreveu um modelo inovador de atendimento integrado para sobreviventes de câncer, denominado de "Modelo Personalizado de Assistência à Sobrevivência ao Câncer". O atendimento era realizado por uma equipe multidisciplinar, em um ambiente de assistência médica coordenado e integrado, para adultos e crianças, para os diversos tipos de cânceres. A coordenaçáo desse serviço é feita com um médico ou enfermeiro especializado, voltada para três níveis, sendo o nível 1 , atendimento primário voltado à prevenção e orientaçôes básicas em saúde; nível 2, com atendimento mais especializado com médicos, enfermeiros oncológicos e atendimento psicossocial, com profissionais especialistas em psicologia; e o nível 3, com atendimento multidisciplinar especializado, incluindo desde médicos pediatras, cardiologistas e dentistas, os quais são voltados para comorbidades fisicas e psicossociais, como hipertensão, hipotiroidismo, depressão, entre outras ${ }^{28}$.

Quanto aos tipos de cânceres inclusos nas redes de apoio para o sobrevivente, este estudo mostra alcance para diversos tipos de cânceres, tendo maior predomínio para o público adulto com câncer de mama e hematológico. Independentemente do tipo de câncer, Campbell et al. ${ }^{19}$ apresentaram em seu estudo a necessidade de definir aspectos importantes do cuidado aos sobreviventes, que inclui o planejamento desses cuidados, pomoção de saúde, apoio e gerenciamento de efeitos tardios do tratamento.

\section{CONCLUSÃO}

As demandas dos sobreviventes envolvem acompanhamento clínico, orientaçôes de cuidados primários, estilo de vida saudável, além de aconselhamento sexual e de fertilidade.

$\mathrm{O}$ atendimento pode ser de forma invidualizada ou em grupo, com base nas diretrizes da ASCO, realizadas em centros oncológicos ou unidades ambulatoriais.

Os sintomas relacionam-se aos domínios físico, psicolólogico e socioecológico, contando com o apoio da equipe multidisciplinar, seja nos cuidados clínicos ou programas educativos. O enfermeiro é o profissional com maior expressão de liderança, tendo sua atuação desde a esfera clínica até a coordenação e atuação quanto ao aconselhamento e orientação para a mudança de comportamentos prejudiciais à saúde.

\section{CONTRIBUIÇÕES}

Andréia Rosangela Delli Paoli Rocha, Erika Yuri Lee, Heloisa Maria Jorviguinoviti dos Reis e Maria das Graças Silva Matsubara contribuíram substancialmente na concepção e/ou planejamento do estudo; na obtenção, análise e/ou interpretação dos dados; na redação e revisão crítica. Edvane Birelo Lopes De Domenico contribuiu na revisão crítica. Todos os autores aprovaram a versão final a ser publicada. 


\section{DECLARAÇÃO DE CONFLITO DE INTERESSES}

Nada a declarar.

\section{FONTES DE FINANCIAMENTO}

Não há.

\section{REFERÊNCIAS}

1. Bray F, Ferlay J, Soerjomataram I, et al. Estatísticas globais de câncer de 2018: estimativas GLOBOCAN de incidência e mortalidade mundial para 36 cânceres em 185 países. CA Cancer J Clin. 2018;68(6):394-424. doi: https://doi.org/10.3322/caac.21492

2. Instituto Nacional de Câncer José Alencar Gomes da Silva. Estimativa 2020: incidência de câncer no Brasil [Internet]. Rio de Janeiro: INCA; 2019 [acesso 2020 ago 20]. Disponível em: https://bit.ly/33AdN8y

3. Rowland JH, Bellizzi KM. Cancer survivorship issues: life after treatment and implications for an aging population. J Clin Oncol. 2014;32(24):2662-8. doi: https://doi.org/10.1200/JCO.2014.55.8361

4. The Economist Intelligence Unit [Internet]. New York: The Economist Intelligence Unit; c2021. Cancer Survivorship country profile Brazil; 2017 [cited 2020 Dez 20]. Available from: https://worldcancerinitiative. economist.com/pdf/BMS-Global-cancer-survivorship/ Brazil.pdf

5. Marzorati C, Riva S, Pravettoni G. Who is a cancer survivor? A systematic review of published definitions. J Cancer Educ. 2017;32(2):228-37. doi: https://doi. org/10.1007/s13187-016-0997-2

6. Ashing-Giwa K, Tapp C, Brown S, et al. Are survivorship care plans responsive to AfricanAmerican breast cancer survivors?: voices of survivors and advocates. J Cancer Surviv. 2013;7(3):283-91. doi: https://doi.org/10.1007/s11764-013-0270-1

7. Cancer.Net [Internet]. Alexandria, VA: American Society of Clinical Oncology; c2005-2021. Survivorship; [cited 20 June 2020]. Available from: http://www.cancer.net/survivorship/what_survivorship

8. Oliveira RAA, Zago MMF, Thorne SE. Interaction between professionals and cancer survivors in the context of Brazilian and Canadian care. Rev LatinoAm Enfermagem. 2017;25:e2972. doi: https://doi. org/10.1590/1518-8345.2253.2972

9. Thorne S, Hislop TG, Kim-Sing C, et al. Changing communication needs and preferences across the cancer care trajectory: insights from the patient perspective. Support Care Cancer. 2014;22(4):100915. doi: https://doi.org/10.1007/s00520-013-2056-4

10. Mayer DK, Deal AM, Crane JM, et al. Using survivorship care plans to enhance communication and cancer care coordination: results of a pilot study. Oncol Nurs Forum. 2016;43(5):636-45. doi: https:// doi.org/10.1188/16.ONF.636-645

11. Darby K, Davis C, Likes W, et al. Exploring the financial impact of breast cancer for African American medically underserved women: a qualitative study. J Health Care Poor Underserved. 2009;20(3):721-8. doi: https://doi.org/10.1353/hpu.0.0176

12. Ganong LH. Integrative reviews of nursing research. Res Nurs Health. 1987;10(1):1-11. doi: https:/doi. org/10.1002/nur.4770100103

13. Aromataris E, Munn Z, editors. JBI manual for evidence synthesis [Internet]. [place unknown]: JBI; 2020. doi: https://doi.org/10.46658/JBIMES-20-0114.

14. Camilo C, Garrido MV. Systematic review in psychology: challenges and guidelines. Anál Psicol. 2019;37(4):535552. doi: https://doi.org/10.14417/ap.1546

15. Santos CMC, Pimenta CAM, Nobre MRC. The PICO strategy for the research question construction and evidence search. Rev Latino-Am Enfermagem. 2007;15(3):508-11. doi: https://doi.org/10.1590/s010411692007000300023

16. Wallace A, Downs E, Gates P, et al. Provision of survivorship care for patients with haematological malignancy at completion of treatment: a cancer nursing practice survey study. Eur J Oncol Nurs. 2015;19(5):516-22. doi: https://doi.org/10.1016/j. ejon.2015.02.012

17. Cox A, Faithfull S. Aiding a reassertion of self: a qualitative study of the views and experiences of women with ovarian cancer receiving long-term nurse-led telephone follow-up. Support Care Cancer. 2015;23(8):2357-64. doi: https://doi.org/10.1007/ s00520-014-2578-4

18. Hall-Alston J. Exercise and the breast cancer survivor: the role of the nurse practitioner. Clin J Oncol Nurs. 2015;19(5):E98-102. doi: https://doi.org/10.1188/15. CJON.E98-E102

19. Campbell G, Thomas TH, Hand L, et al. Caring for survivors of gynecologic cancer: assessment and management of long-term and late effects. Semin Oncol Nurs. 2019;35(2):192-201. doi: https://doi. org/10.1016/j.soncn.2019.02.006

20. Heveran L, Andrewes T. Nursing measures to support the needs of haematological cancer survivors post-treatment: a literature review. Br J Nurs. 2020;29(3):S18-S24. doi: https://doi.org/10.12968/bjon.2020.29.3.S18

21. Karvinen KH, Bruner B, Truant T. Lifestyle counseling practices of oncology nurses in the United States and Canada. Clin J Oncol Nurs. 2015;19(6):690-6. doi: https://doi.org/10.1188/15.CJON.690-696

22. Ferguson J, Aning J. Prostate cancer survivorship: a nurse-led service model. Br J Nurs. 2015;24(18):S14-21. doi: https://doi.org/10.12968/bjon.2015.24.Sup18.S14 
23. Singh-Carlson S, Wong F, Oshan G. Evaluation of the delivery of survivorship care plans for South Asian female breast cancer survivors residing in Canada. Curr Oncol. 2018;25(4):e265-e274. doi: https://doi.org/10.3747/ co. 25.3734

24. Nolte L, Kinnane N, Lai-Kwon J, et al. The impact of survivorship care planning on patients, general practitioners, and hospital-based staff. Cancer Nurs. 2016;39(6):E26-E35. doi: https://doi.org/10.1097/ NCC.0000000000000329

25. Corcoran S, Dunne M, McCabe MS. The role of advanced practice nurses in cancer survivorship care. Semin Oncol Nurs. 2015;31(4):338-347. doi: https:// doi.org/10.1016/j.soncn.2015.08.009

26. Peixoto NMSM, Peixoto TASM, Pinto CAS, et al. Estratégias de autogestão da ansiedade nos sobreviventes de cancro: revisão sistemática da literatura. Rev Enf. 2017;4(12):143-54. doi: http://doi.org/10.12707/ RIV16024

27. Oliveira RAA, Conceição VM, Araujo JS, et al. Concept analysis of cancer survivorship and contributions to oncological nursing. Int J Nurs Pract. 2018;24(1). doi: http://doi.org/10.1111/ijn.12608

28. Loonen JJ, Blijlevens NMA, Prins J, et al. Cancer survivorship care: person centered care in a multidisciplinary shared care model. Int J Integr Care. 2018;18(1):4. doi: http://doi.org/10.5334/ijic.3046

29. Kinahan KE, Kircher S, Altman J, et al. Promoting the shared-care model for adolescent and young adults with cancer: optimizing referrals and care coordination with primary care providers. J Natl Compr Canc Netw. 2017;15(1):38-44. doi: http://doi.org/10.6004/ jnccn.2017.0005

30. Peckham J, Mott-Coles S. Interprofessional lung cancer tumor board: the role of the Oncology Nurse Navigator in improving adherence to national guidelines and streamlining patient care. Clin J Oncol Nurs. 2018;22(6):656-62. doi: http://doi.org/10.1188/18. CJON.656-662

31. Handberg C, Thorne S, Maribo T. When a policy decision meets practice realities: the case of cancer survivorship care and rehabilitation needs assessment. Eur J Oncol Nurs. 2018;33:70-77. doi: http://doi. org/10.1016/j.ejon.2018.01.010

32. Pandey P, Barber F. Improving the education and training of primary care nurse practitioners to meet the healthcare needs of long-term cancer survivors. Clin J Oncol Nurs. 2016;20(3):334-5. doi: http://doi.org/10.1188/16. CJON.334-335
33. Hartnett E. Impact of collaborative evidence-based breast cancer survivorship care guidelines on survivors and providers. Clin J Oncol Nurs. 2016;20(3):332-3. doi: http://doi.org/10.1188/16.CJON.332-333

34. Ashing K, Serrano M, Weitzel J, et al. Towards developing a bilingual treatment summary and survivorship care plan responsive to Spanish language preferred breast cancer survivors. J Cancer Surviv. 2014;8(4):580-94. doi: http:// doi.org/10.1007/s11764-014-0363-5

35 . Building a bridge of continued care for cancer survivors. J Oncol Pract. 2006;2(2):77-82. doi: 10.1200/ JOP.2006.2.2.77

36. Vidotti JF, Scorsolini-Comin F, Santos MA. Qualidade de vida em sobreviventes de longo prazo ao câncer de mama: análise da produção científica. Psicol Teor Prat. 2013;15(3):49-68.

37. Tompkins C, Scanlon K, Scott E, et al. Survivorship care and support following treatment for breast cancer: A multi-ethnic comparative qualitative study of women's experiences. BMC Health Serv Res. 2016;16:401. doi: http://doi.org/10.1186/s12913-016-1625-x

38. Seiler A, Jenewein J. Resilience in cancer patients. Front Psychiatry. 2019;10:208. doi: https://doi.org/10.3389/ fpsyt.2019.00208

39. Ruini C, Vescovelli F, Albieri E. Post-traumatic growth in breast cancer survivors: new insights into its relationships with well-being and distress. J Clin Psychol Med Settings. 2013;20(3):383-91. doi: https://doi.org/10.1007/ s10880-012-9340-1

40. Post KE, Moy B, Furlani C, et al. Survivorship Model of Care: Development and implementation of a nurse practitioner-led intervention for patients with breast cancer. Clin J Oncol Nurs. 2017;21(4):E99-E105. doi: https://doi.org/10.1188/17.CJON.E99-E105

Recebido em 18/1/2021 Aprovado em 12/5/2021

Editor-associado: Fernando Lopes Tavares de Lima. Orcid iD: https://orcid.org/ 0000-0002-8618-7608 Editora-científica: Anke Bergmann. Orcid iD: https://orcid.org/0000-0002-1972-8777 